\title{
HIGH RESOLUTION STUDIES OF COMPACT BINARIES IN GLOBULAR CLUSTERS WITH HST AND ROSAT
}

\author{
JONATHAN E. GRINDLAY \\ Harvard-Smithsonian Center for Astrophysics \\ 60 Garden Street \\ Cambridge, MA 02138, USA
}

\begin{abstract}
The studies of compact binaries containing an accreting white dwarf or neutron star in the dense cores of globular clusters have made considerable progress in the past few years as a result of the high resolution images obtained with HST and ROSAT. It is now clear that cluster cores contain a significant population of these systems which must constrain the similarly large populations of millisecond pulsars as well as dynamical histories of clusters. The population of $\operatorname{dim} \mathrm{x}$-ray sources appears to be dominated by cataclysmic variables (CVs) formed by tidal capture and not exchange collisions. Our recent HST/FOS spectra of the first CVs in a cluster core, summarized here in more detail, suggest that cluster cores may contain a significant population of magnetic CVs. The required magnetic WDs may arise in spun-up cores of blue stragglers.
\end{abstract}

\section{Introduction}

The cores of globular clusters have been long recognized as the site of stellar encounters leading to binary production (and destruction) and the dynamical evolution of the entire cluster (cf. Hut et al 1992 for a general review). Indeed, even the evolution of the globular cluster system in the Galaxy is driven to some extent by the stellar dynamics, and compact binary content, of the cluster core with core collapse apparently more likely to occur in disk clusters subject to tidal shocking. One of the most direct probes of compact binaries in cluster cores are the dim x-ray sources (Grindlay 1994 and references therein), which were discovered in the first imaging $x$-ray survey of globulars (Hertz and Grindlay 1983; HG) and have now been seen 
in much greater numbers (at least 27; cf. Johnston, Verbunt and Hasinger 1996) with more sensitive ROSAT observations. The other major tool is of course HST, for which the unprecedented spatial resolution has allowed both emission line object imaging searches and spectroscopic identification of both dim sources and compact binaries generally deep within the cores of core collapsed globulars.

In this paper we summarize first the increasingly rich studies of compact binaries in globulars being carried out with HST and then the ROSAT data and the possible dependences on cluster parameters of the integrated core $\mathrm{x}$-ray luminosities for the dim sources. We provide more details for our recent HST spectra of the first CVs, or (more exactly) CV candidates, in the core of the core collapsed cluster NGC 6397 together with a first look at our very deep ROSAT HRI observation of the same cluster. At least 2-3 additional dim sources, for a total of 5-6, are now detected within the central $\sim 10^{\prime \prime}$ of the cluster center. Our HST/FOS optical spectra of the three brightest candidates suggest these CVs are magnetic, or of the DQ Her type. This would suggest that magnetic white dwarfs (WDs) are somehow enhanced in globular clusters over their relative abundance in the field. We outline a possible model for the production of magnetic WDs in globulars as the WD remnants of rapidly rotating cores of blue stragglers.

\section{HST Studies of Compact Binaries in Globular Cluster Cores}

\subsection{WFPC1 AND WFPC2 OBSERVATIONS OF NGC 6397 AND NGC 6752}

In order to search for CVs in globulars to test the original proposal that they account for the (vast) majority of dim x-ray sources in clusters (HG, Grindlay 1994), we have developed and conducted a photometric imaging search for $\mathrm{H} \alpha$ emission line objects in cluster cores using the WFPC1 and WFPC2 cameras on HST. All classes of CVs show $\mathrm{H} \alpha$ in emission (except for some dwarf novae during outburst maxima, when the line can go into absorption) so that emission lines rather than blue continuum colors (though this is also checked) or variability are our primary search criterion for selection of CV candidates. The implementation of this narrow-band photometry with HST using DAOPHOT as well as the calibrations and uncertainties are described in detail by Cool (1993).

Our WFPC1 results for the nearby core collapse cluster NGC 6397 are presented by Cool et al (1995; CG95). Three significant detections of $\mathrm{H} \alpha$ bright objects were found in the error circles ( $\sim 6^{\prime \prime}$ radius) of the three brightest dim $\mathrm{x}$-ray sources in the cluster core discovered with ROSAT (Cool et al 1993; CG93). The three objects have apparent $\mathrm{R}$ magnitudes of $17.8,18.8$ and 19.5 corresponding to absolute magnitudes $\mathrm{M}_{R}=5.6,6.6$ and 7.3. Thus all three are typical of nova-like CVs and not dwarf novae 
(in outburst) and yet are still somewhat brighter than the median absolute magnitude $(\sim 8)$ of disk CVs. All three were also uv bright, as verified from comparison with archival FOC images taken in the uv (cf. CG95). In addition there were three additional possible CV candidates which showed either possible $\mathrm{H} \alpha$ emission or uv excess or both. One or more of these may be now identified with still fainter ROSAT sources as discussed below.

In NGC 6752, we obtained WFPC1 images in our first exploratory $\mathrm{H} \alpha$ search with HST (in Cycle 1$)$. The relatively shallow exposures $(6 \times$ $1300 \mathrm{~s}$ in $\mathrm{H} \alpha ; 2 \times 500 \mathrm{~s}$ in $\mathrm{R}$ ) only reached equivalent absolute magnitudes $\mathrm{M}_{R} \sim 5$ for the detection of $\mathrm{H} \alpha$ bright objects (Cool 1993) and was thus not sensitive enough to see CVs except dwarf novae in outburst. However in deeper Cycle 5 observations with WFPC2 (cf. Bailyn et al 1996), we have found two $\mathrm{H} \alpha$ objects in the central core (within the PC chip only). One of these CV candidates is in the error circle of one of the two (brightest) dim sources in the core (Grindlay 1993a, Grindlay and Cool 1996).

\subsection{FOS SPECTRA OF CV CANDIDATES IN NGC 6397}

We have recently reported (Grindlay et al 1995; GC95) the first spectra of $\mathrm{CV}$ candidates (or any stars below the ms turnoff) in the central core of a globular cluster, NGC 6397.
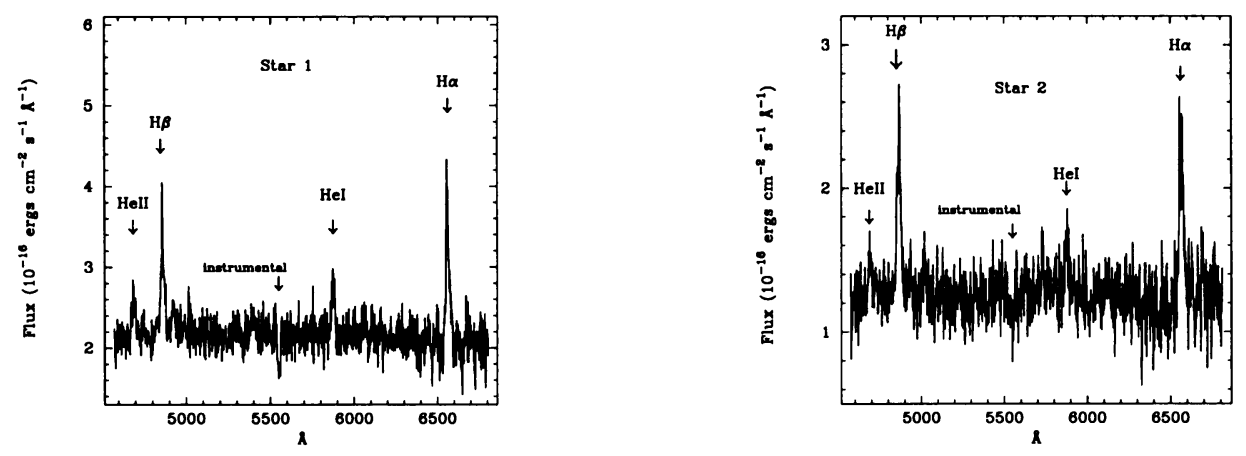

Figure 1. HST/FOS spectra of star 1 (left) and 2 (right) in NGC 6397.

We observed with the HST/FOS on UT 2 April 1995 the three brightest $\mathrm{H} \alpha$ emission line candidates discovered with our WFPC1 photometry (CG95). Spectra were obtained not only to confirm the suspected $\mathrm{H} \alpha$ emission but to further test whether these objects are indeed CVs or could be quiescent LMXBs (see below). The FOS spectra are all remarkably similar (cf. Figure 1) and show not only the bright Balmer lines but also He I and He II emission as is often detected in magnetic CVs of the DQ Her type (cf. Patterson 1994). 
At least some of the Balmer emission lines and He II line are doublepeaked (cf.Figure 2), suggesting these objects undoubtedly contain accretion disks with characteristic velocity half-widths of $\sim 600 \mathrm{~km} / \mathrm{s}$ (cf. GC95), or Keplerian radii (for the emission line regions, and an assumed $1 M_{\odot}$ compact object) of $\sim 3 \times 10^{10} \mathrm{~cm}$. This in turn suggests a minimum orbital period for the probable binary companions in these systems of $\sim 2.4$ hours if their disks extend out to $\sim 0.5$ their binary companion separations as is typical in compact binaries. These binaries could, therefore, be very compact though probably not as compact as AM Her type CVs which have an orbital period distribution peaked at $\lesssim 2$ hours.

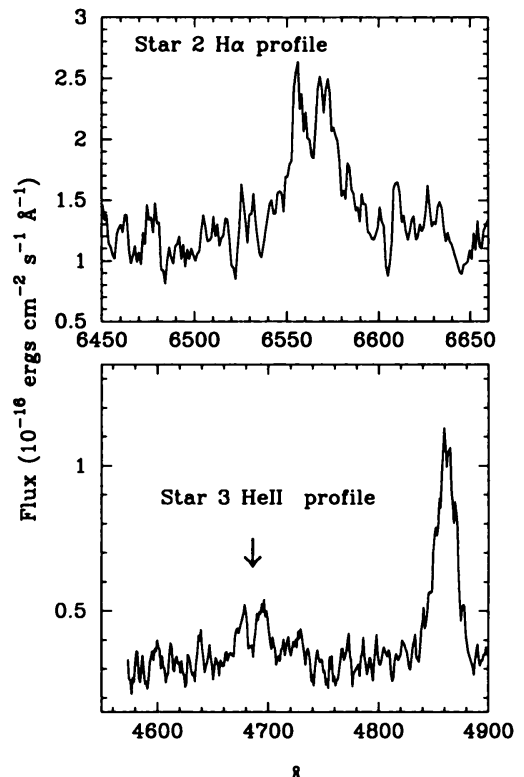

Fig. 2: Double-peaked emission profiles.

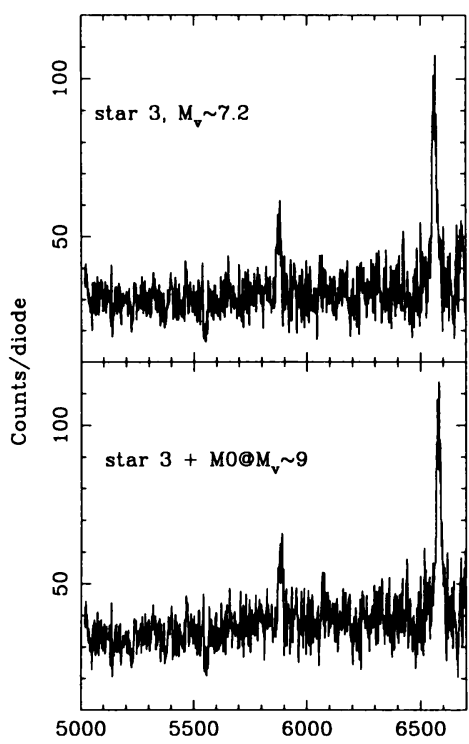

Fig. 3: Star 3 (top) vs. simulated spectrum including $\mathrm{M} 0$ star

A crude upper limit for the binary periods $(\lesssim 6 \mathrm{~h})$ can be estimated from the fact that the secondary mass must be $\lesssim 0.45 M_{\odot}$ due to the absence of any detectable absorption lines or red continuum in the spectra (GC95), as shown in Figure 3 . The simulated spectrum (bottom) of star 3 with a M0 companion (with $M_{V} \sim 9$ ) shows a readily detectable red continuum not seen in the actual data (top), thus constraining the secondary mass for at least this faintest of our three CV candidates in NGC 6397.

\subsection{DWARF NOVAE SEARCHES WITH HST}

Searches for variable, and uv-bright, objects have now been conducted in several globulars to measure the number of dwarf novae. Paresce and De Marchi (1994) have found (just) one DN in the core of 47 Tuc, and indeed 
a repeat outburst of this same object was found (and no others) in more extensive coverage of the cluster core by Shara and collaborators (these proceedings). A search of a portion ( 0.25) of the central core of NGC 6752 has also been reported by Shara et al (1995); no CV candidates (or DN) were found suggesting that CVs may be depleted relative to model expectations (and see Shara, these proceedings). Whereas these studies are indisputable in their sensitivity to DN outbursts, which could not be missed, they are less restrictive in their sensitivity to CVs in quiescence since their sensitivity to the (often) modest variability ( $\lesssim 0.1 \mathrm{mag}$ ) and their relatively non-uniform sampling have limited their sensitivity to CVs overall.

\section{ROSAT Studies of Compact Binaries in Globular Cluster Cores}

As with HST, the ability to measure high resolution $\mathrm{x}$-ray images of clusters has proven crucial to the identification and study of compact $x$-ray binaries in clusters. However, the best $\mathrm{x}$-ray imaging resolution thus far - the HRI on ROSAT, with $\sim 3$ " resolution is of course still worse than even groundbased optical images. This is largely (but not totally) compensated by the enormous difference in source spatial density: at the faintest soft $\mathrm{x}$-ray luminosities thus far reached $\left(\mathrm{L}_{x} \sim 2 \times 10^{31} \mathrm{erg} / \mathrm{s}\right)$, the highest density of discrete sources previously reported is the $\sim 3-4$ sources within a $10^{\prime \prime}$ radius of the center of NGC 6397 (CG93). However it is clear that even more sources exist in the cores of high density clusters like 47 Tuc, where Hasinger et al (1994) find at least 4-9 discrete sources (with at least 4 in the core) superimposed on diffuse emission in the cluster core that very likely is due to a much larger underlying source population. We reported (CG93) a similar effect in NGC 6397 and now find (see below) at least 2-3 additional dim sources, for a total of $5-6$, in just the cluster core region so that the total population is very much confusion limited even for the ROSAT HRI. Thus a true census of the cluster dim source population must await the significantly higher spatial resolution $\left(\sim 0.5^{\prime \prime}\right)$ of AXAF.

\subsection{INTEGRATED X-RAY LUMINOSITIES OF GLOBULAR CORES}

Despite the need for very high spatial resolution to study the entire dim source population, studies of globulars with the lower spatial resolution $\left(\sim 30^{\prime \prime}\right)$ PSPC detector on ROSAT can measure the total integrated $\mathrm{x}$ ray luminosity of compact binaries in cluster cores (e.g. Johnston et al 1994, 1996). A survey of 9 globulars with large core radii gave detections of integrated core luminosities (within $\sim 3 \mathrm{r}_{c}$ of the cluster centers; denoted here as "extended core") of $\mathrm{L}_{x, \text { core }} \sim 1.5$ and $0.5 \times 10^{32} \mathrm{erg} / \mathrm{s}$ for NGC 6366 and NGC 6809 (M55), respectively and upper limits typically $\lesssim 1 \times$ $10^{32} \mathrm{erg} / \mathrm{s}$ for the remaining 7 clusters (Johnston et al 1996). A complete 
list of all currently measured values of $\mathrm{L}_{x, \text { core }}$ (given in erg/s in the ROSAT band, $0.2-2 \mathrm{keV}$ and from HG, CG93, Grindlay 1993a, Hasinger et al 1994, Rappaport et al 1994 and Johnston et al 1994, 1996) for all 14 globulars detected is given below in Table 1.

Table 1: Globular Cluster Dim Source Luminosity vs. T2 or T3 prediction

\begin{tabular}{|rrrrrrr|}
\hline & & \multicolumn{3}{c}{ Pryor/Meylan: } & \multicolumn{2}{c|}{ Djorgovski: } \\
Cluster & {$\left[\mathrm{F}_{e} / \mathrm{H}\right]$} & $\log \left(\mathrm{L}_{x, \text { core }}\right)$ & $\log (\mathrm{T} 2)$ & $\log (\mathrm{T} 3)$ & $\log (\mathrm{T} 2)$ & $\log (\mathrm{T} 3)$ \\
\hline NGC104 & -0.71 & 33.6 & 7.73 & 5.18 & 7.64 & 5.24 \\
1904 & -1.69 & 33.7 & 6.75 & 4.65 & 6.62 & 4.62 \\
5139 & -1.59 & 32.6 & 7.51 & 5.76 & 6.91 & 5.31 \\
6304 & -0.59 & 33.0 & - & - & 6.98 & 4.73 \\
6341 & -2.24 & 32.5 & 6.86 & 4.66 & 6.56 & 4.96 \\
6366 & -0.99 & 32.2 & 4.77 & 3.82 & 5.48 & 4.33 \\
6397 & -1.91 & 32.5 & 6.95 & 4.30 & 6.28 & 3.43 \\
6541 & -1.83 & 33.2 & 7.69 & 4.94 & 7.37 & 5.12 \\
6626 & -1.44 & 32.8 & 7.49 & 5.04 & 7.33 & 4.74 \\
6656 & -1.75 & 32.3 & 7.29 & 5.29 & 6.72 & 4.87 \\
6752 & -1.54 & 32.6 & 6.99 & 4.39 & 7.14 & 4.69 \\
6809 & -1.82 & 31.8 & 6.26 & 5.01 & 5.25 & 4.20 \\
7099 & -2.13 & 32.7 & 7.39 & 4.44 & 6.72 & 4.17 \\
PAL 2 & -1.68 & 34.0 & - & - & 7.64 & 5.39 \\
\hline
\end{tabular}

As frequently pointed out (e.g. Hut et al 1992, CG95), 2-body ("T2") tidal capture production of the dim sources (as for the luminous LMXBs) would predict a total number of sources scaling as $\rho_{c} \mathrm{M}_{c} / \mathrm{v}_{c}$, where the c subscript refers to core values and the $\rho$ and $\mathrm{M}$ are cluster core density and total mass, and $\mathrm{v}$ is the central velocity dispersion. Since $\mathrm{M}_{c} \propto \rho_{c}$, this then gives a scaling as $\rho_{c}^{2}$. A flatter dependence on $\rho_{c}\left(\propto \rho_{c}{ }^{1 / 2} M_{c}\right)$ is found for millisecond pulsars (cf. Johnston et al 1992) which has usually been interpreted that they can form in lower density clusters by 3-body ("T3") exchange collisions of NSs into primordial binaries which can have longer periods in low density clusters without being disrupted. Thus in Table 1, we also tabulate for each cluster the quantities $\log (\mathrm{T} 2) \propto \rho_{c}^{2} \mathrm{r}_{c}^{3}$ $/ \mathrm{v}_{c}$ and $\log (\mathrm{T} 3) \propto \rho_{c}^{1.5} \mathrm{r}_{c}^{3} / \mathrm{v}_{c}$, where the cluster parameters for $\rho_{c}$ and $\mathrm{r}_{c}$ have been taken from Djorgovski (1993) or Pryor and Meylan (1993) and the central velocity dispersions from Pryor and Meylan (1993). Total dim source numbers may be estimated from the extended core luminosities only if the dim source luminosity function is known.

The deepest observations (NGC 6397; see below) suggest the number of dim sources increases down to luminosities of at least as low as $\mathrm{L}_{*} \sim 2$ $\mathrm{x} 10^{31} \mathrm{erg} / \mathrm{s}$ and with a power law index $\alpha \gtrsim 1$ for an integral number of sources $\mathrm{N}_{\operatorname{dim}}\left(\gtrsim \mathrm{L}_{x}\right)=\mathrm{A} \cdot\left(\mathrm{L}_{x} / \mathrm{L}_{*}\right)^{-\alpha}$, where $\mathrm{A}$ is the normalization factor 
for which the dependence $\mathrm{A}\left(\rho_{c}, \mathrm{M}_{c}, \mathrm{v}_{c}\right)$ is desired. Thus the extended core luminosity may be obtained by integrating the above integral luminosity function over $\mathrm{L}_{x}$ (equivalent to integrating $\left(\mathrm{dN} / \mathrm{dL}_{x}\right) \cdot \mathrm{L}_{x} \mathrm{dL}_{x}$ ) so that $\mathrm{L}_{x, \text { core }}=\mathrm{C} \cdot \mathrm{A} \cdot\left(\mathrm{L}_{x} / \mathrm{L}_{*}\right)^{-\alpha+1}$, where $\mathrm{C}$ is the integration constant. Thus for $\alpha \sim 1$, and assuming all clusters have the same luminosity function and thus value of $\alpha$ (which may also depend on the same cluster properties as does $\mathrm{A})$, the normalization constant $\mathrm{A}\left(\rho_{c}, \mathrm{M}_{c}, \mathrm{v}_{c}\right) \propto \mathrm{L}_{x, \text { core }}$. The values of $\mathrm{L}_{x, \text { core }}$ given in Table 1 thus trace the dim source content.

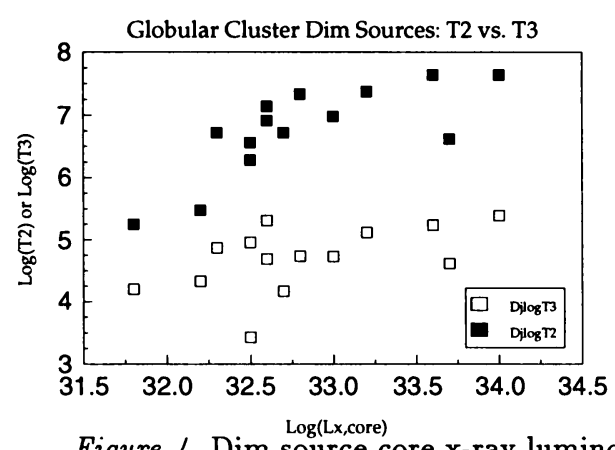

Figure 4. Dim source core x-ray luminosity vs. T2 or T3 scaling.

In Figure 4 we plot the values of $\log (\mathrm{T} 2)$ and $\log$ (T3) (using the Djorgovski 1993 values) vs. $\log \mathrm{L}_{x, \text { core }}$ for the currently known cluster dim source population. It is clear that the $\log (\mathrm{T} 3)$ scaling is much flatter (particularly for $\mathrm{L}_{x, \text { core }} \lesssim 10^{33} \mathrm{erg} / \mathrm{s}$ ) than the $\log (\mathrm{T} 2)$ values, or that the total (integrated) core luminosity does not depend on $\log (\mathrm{T} 3)$ but rather $\log (\mathrm{T} 2)$. Similar results, but with larger scatter, are found for the Pryor-Meylan values for T2 and T3. Thus the total number of cluster dim sources, and thus cluster CVs, appears to be better described by tidal capture formation than by exchange collisions between cluster WDs and primordial binaries.

We also list in Table 1 the cluster metallicities $[\mathrm{Fe} / \mathrm{H}]$ (from Djorgovski 1993). These show that in contrast to the 12 clusters containing the luminous LMXBs, which are significantly metal rich (Grindlay 1993b) with $<[\mathrm{Fe} / \mathrm{H}]>\sim-1.17$ (Bellazzini et al 1995), the dim source clusters have mean $\langle[\mathrm{Fe} / \mathrm{H}]>\sim-1.57$, or approximately that of the cluster population at large. Thus, if the larger $[\mathrm{Fe} / \mathrm{H}]$ for the LMXB globulars suggests they have flatter IMFs and an enhanced NS population, the (massive) WDs needed for the dim sources are a more constant fraction of cluster mass.

\subsection{DEEP ROSAT OBSERVATION OF NGC 6397}

Over the time period 17 March - 2 April, 1995, we obtained a deep (75 ksec) HRI exposure on NGC 6397. By good fortune the final 12 hours (on 
April 2) overlapped with our HST/FOS exposures. Detailed analysis of this rich data set is in progress; space limitations do not permit presentation of images here. Qualitatively, the image looks similar to our $18 \mathrm{ksec}$ image (CG93) recorded in two epochs (March 1991 and March 1992) although we detect additional emission from one or more sources in the vicinity of $\mathrm{B}$ and several in the C1-C3 complex. Source A is fainter and near the detection limit of $\mathrm{L}_{x} \sim 1 \times 10^{31} \mathrm{erg} / \mathrm{s}$, and a new source ("D") is detected $\sim 1^{\prime} \mathrm{NE}$ of the core. Of greatest interest is the additional structure now seen in the central $10-20$ " of the core, where fainter sources possibly consistent with the $\mathrm{H} \alpha$ candidate stars 6 and 7 of CG95 are seen. The entire central source complex (5-6 sources) is clearly variable, with different sources dominating over the 2 week observation interval. Statistics limit short-time variability and comparison with the time-resolved FOS spectra. The new sources are all fainter than the original $\mathrm{C} 1-\mathrm{C} 3$, indicating the luminosity function continues to rise down to at least $\mathrm{L}_{x} \sim 2 \times 10^{31} \mathrm{erg} / \mathrm{s}$.

\section{Nature of the CVs in Globulars: Magnetics ?}

The presence of the He I and He II emission lines in the spectra of the first three CVs measured in the core of a globular cluster is striking. While occasionally dwarf novae or nova-likes show He emission lines, this is much more common in the magnetic CVs. The He lines are not strong enough (and the absolute magnitudes are in any case too bright) to be AM Her types, but the spectra are not dissimilar from the lower magnetic field IP class objects (e.g. Singh et al 1995), which have ratios of $\mathrm{EW}(\mathrm{He} \mathrm{II}) / \mathrm{EW}(\mathrm{H} \beta) \sim 0.3$ 0.5, or quite similar to the values for stars 1-3 (cf. GC95). However, given the direct evidence for accretion disks in the cluster objects, they most closely resemble DQ Her type CVs with still lower fields. This class is also favored since they generally obey the correlation between $\mathrm{x}$-ray/optical flux continuum ratio vs. $\mathrm{EW}(\mathrm{H} \beta$ ) for CVs (cf. Patterson and Raymond 1985; PR) whereas the AM Her systems do not. As shown in the accompanying paper by Grindlay and Cool, the now-measured $\mathrm{EW}(\mathrm{H} \beta)$ and visual flux (from spectral continuum) values put the three objects even closer to the $\mathrm{PR}$ correlation and distinct from the quiescent LMXB Cen X4.

The fraction of CVs in the field that are DQ Her type is not well known but is probably $\lesssim 0.25$ (cf. review by Patterson 1994). Thus finding (possibly) 3 for 3 in a globular would seem unlikely (binomial probability $\lesssim 0.02$ ). Why should there be more magnetic systems or at least magnetic white dwarfs (WDs) in globulars ? Prompted by the results for blue straggler formation presented by Rasio at this meeting (cf. these proceedings and Lombardi et al 1995), we propose the following heuristic model (or scenario) for enhanced magnetic CV formation. The basic idea is to use the 
WD remnants from blue straggler stars (BSS) formed by the encounters of two stars each near the turnoff mass (or with one already a sub-giant). Such stars, with radiative cores and convective envelopes, will have rapidly rotating cores after the BSS formation. However, as Lombardi et al point out, their convective envelopes will be slowed by magnetic braking on timescales short enough $\left(\sim 10^{5}\right.$ years $)$ to account for the lack of rapid rotation typically observed in BSS. This combination of a rapidly rotating core and differentially (more slowly) rotating envelope must be an ideal dynamo generator: at least it contains the requisite ingredients of (rapid) differential rotation and convection. Therefore, we propose that the cores of BSS, which will evolve to WDs, are likely to have greater magnetic fields than main sequence stars of comparable total mass and thus preferentially produce magnetic WDs.

When captured by lower mass main sequence stars (with mass ratios $\mathrm{q} \lesssim 0.8$ for stable mass transfer subsequently), these magnetic WDs will then give rise to magnetic CVs. Since these WDs are produced by stars of typically twice the current turnoff mass they will be more massive than "currently produced" WDs and thus favored for CVs. Furthermore, since Proctor, Bailyn and Demarque (1995) show that the colors of the BSS in NGC 6397 require that the Lombardi et al results, in which the merged stars are predicted to be unmixed, must be modified by either subsequent rapid mixing or the merger of 3 stars into a BSS (as from a star+binary merger), then if magnetic WDs are preferentially produced in spun-up cores of of BSS, they are again more likely to form observable CVs. Since in a collapsed core like NGC 6397 an appreciable fraction of the stars above the turnoff are BSS (some 9 of the $\sim 60$ stars above the turnoff within our (CG95) central $38 \times 35$ arcsec WFPC1 frame are BSS), the penalty for having to undergo two collision or capture processes (to first form a BSS and then have the massive WD captured into a CV) is not overwhelming. The observed BSS fraction, combined with the shorter BSS lifetimes, suggests that perhaps $25 \%$ of the post-main sequence stars in the central region of NGC 6397 could be processed through BSS so that the production of massive WDs at the current epoch is significantly enhanced over the baseline production from massive (e.g. $\gtrsim 2 M_{\odot}$ ) progenitors if the mass function $\mathrm{dN} / \mathrm{dm} \propto$ $\mathrm{m}^{-(1+\eta)}$ has index $\eta \gtrsim 1$.

\section{Conclusions}

We conclude that the dim x-ray sources in globulars are primarily CVs formed by tidal capture. The CVs may contain a significantly enhanced magnetic fraction which could arise from production of magnetic, and more massive, WDs in the spun-up cores of BSS produced in dense cluster cores. 
The lack of significant metallicity enhancement of the 14 clusters in which dim sources have thus far been detected suggests that the massive WDs likely incorporated into the cluster dim sources are distributed more uniformly (perhaps from BSS) than are the corresponding NSs in the LMXB clusters. Additional HST imaging and spectra, as well as higher sensitivity and resolution (AXAF) x-ray observations, can provide rigorous tests of this picture.

I thank Adrienne Cool for discussions. This work was partially supported by NASA grants NAGW-3280 and HST grant GO-5497.

\section{References}

Bailyn, C. et al 1996, in preparation

Bellazzini, M. et al 1995, ApJ, 439, 687

Cool, A.C. 1993, Ph.D. Thesis, Harvard University

Cool, A. et al 1993, ApJ, 410, L103 (CG93)

Cool, A. et al 1995, ApJ, 439, 695 (CG95)

Djorgovski, S. 1993 in Dynamics of Globular Clusters (S. Djorgovski and G. Meylan, eds.), ASP Conf. Series, Vol. 50, p. 373

Grindlay, J.E. 1993a, in Dynamics of Globular Clusters (S. Djorgovski and G. Meylan, eds.), ASP Conf. Series, Vol. 50, p. 285

Grindlay, J.E. 1993b, in The Globular Cluster-Galaxy Connection, (G. Smith and J. Brodie, eds.), ASP Conf. Series, Vol. 48, p. 156

Grindlay, J.E. 1994, in Evolution of X-Ray Binaries, (S. Holt and C. Day, eds.), AIP Conf.Proc., 308, 339

Grindlay, J. and Cool, A. 1996, in preparation

Grindlay, J., Cool, A. et al 1995, ApJ, 455, L47 (GC95)

Hasinger, G.. Johnston, H. and Verbunt, F. 1994, A\&A, 288, 466

Hertz, P., and Grindlay, J.E. 1983, ApJ, 278, 137 (HG)

Hut, P. et al 1992, PASP, 104, 981

Johnston, H. et al 1992, in X-ray Binaries and Millisecond Pulsars (E. Van den Heuvel and S. Rappaport, eds.), Kluwer, Dordrecht, p. 349

Johnston, H., Verbunt, F. and Hasinger, G. 1994, A\&A, 289, 763

Johnston, H., Verbunt, F. and Hasinger, G. 1996, A\&A, in press.

Lombardi, J. , Rasio, F. and Shapiro, S. 1995, ApJ, 445, 117

Paresce, F. and DeMarchi, G. 1994, ApJ, 427, L33

Patterson, J. 1994, PASP, 106, 209

Patterson, J. and Raymond, J. 1985, ApJ, 292, 535 (PR)

Proctor, A, Bailyn, C. and Demarque, P. 1995, ApJ, submitted

Pryor, C. and Meylan, G. 1993 in Dynamics of Globular Clusters (S. Djorgovski and G. Meylan, eds.), ASP Conf. Series, Vol. 50, p. 357

Rappaport, S. et al 1994, ApJ, 423, 633

Shara, M., Drissen, L., Bergeron, L., and Paresce, F. 1995, ApJ, 441, 617

Singh, K. et al 1995, ApJ, 453, L95 